\title{
Article
}

\section{Emergence of transient domain wall skyrmions after ultrafast demagnetization}

\author{
Lepadatu, Serban
}

Available at https://clok.uclan.ac.uk/34759/

Lepadatu, Serban orcid iconORCID: 0000-0001-6221-9727 (2020) Emergence of transient domain wall skyrmions after ultrafast demagnetization. Physical Review B, 102 (9). 094402. ISSN 2469-9950

It is advisable to refer to the publisher's version if you intend to cite from the work. http://dx.doi.org/10.1103/PhysRevB.102.094402

For more information about UCLan's research in this area go to http://www.uclan.ac.uk/researchgroups/ and search for < name of research Group>.

For information about Research generally at UCLan please go to http://www.uclan.ac.uk/research/

All outputs in CLoK are protected by Intellectual Property Rights law, including Copyright law. Copyright, IPR and Moral Rights for the works on this site are retained by the individual authors and/or other copyright owners. Terms and conditions for use of this material are defined in the policies page.

\section{CLoK}

Central Lancashire online Knowledge www.clok.uclan.ac.uk

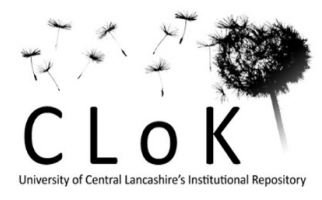




\title{
Emergence of transient domain wall skyrmions after ultrafast demagnetization
}

\author{
Serban Lepadatu $\odot^{*}$ \\ Jeremiah Horrocks Institute for Mathematics, Physics and Astronomy, University of Central Lancashire, Preston PR1 2HE, United Kingdom
}

(Received 7 June 2020; accepted 18 August 2020; published 1 September 2020)

\begin{abstract}
It is known that ultrafast laser pulses can be used to deterministically switch magnetization and create skyrmions; however, the deterministic creation of a single Néel skyrmion after ultrafast demagnetization remains an open question. Here we show domain wall skyrmions also emerge in systems with broken inversion symmetry after exposure to an ultrafast laser pulse, carrying an integer topological charge. While domain wall skyrmions do not appear in the relaxed state due to quick thermal decay following an Arrhenius law, they play a key role in controlling the final skyrmion population through annihilations with skyrmions of opposite topological charge, with the resultant skyrmion states following a Poisson distribution. Using single-shot linearly polarized laser pulses, as well as a train of circularly polarized laser pulses, we show that when a high degree of disorder is created, the possibility of nucleating a single Néel skyrmion is accompanied by the possibility of nucleating a skyrmion with domain wall skyrmion pair, which results in a self-annihilation collapse.
\end{abstract}

DOI: 10.1103/PhysRevB.102.094402

\section{INTRODUCTION}

The Dzyaloshinskii-Moriya interaction (DMI) [1,2] enables formation of a wide range of exciting magnetic objects which are topologically stabilized, and belong to a wider class of topological magnetic solitons [3]. The most widely studied topological object is the skyrmion [4], which has been observed both in materials with bulk DMI [5,6], and ultrathin films with interfacial DMI $[7,8]$. The topological charge is defined in Eq. (1), and for a skyrmion it takes on unit values [9]. Such objects are intensely studied due to the ability to manipulate them with electrical currents through interfacial spin-orbit torques [10-12], bulk spin-transfer torques [13], as well as interfacial spin-transfer torques [14]. Skyrmions have also been proposed in antiferromagnetic materials [15], being a type of two-dimensional antiferromagnetic topological soliton [16], and could potentially be used as information carriers in antiferromagnetic spintronics [17]. Other related topological structures include antiskyrmions [18,19], skyrmioniums [20], or more generally skyrmion bags [21]. Another type of object with unit topological charge is the domain wall (DW) skyrmion, which following initial theoretical studies [22-24] has been revisited recently [25]. The DW skyrmion occurs in Néel domain walls as a $360^{\circ}$ transverse rotation of magnetization, and is stabilized by the interfacial DMI. While such structures have only very recently been observed experimentally [26], we show here they are ubiquitous in magnetization recovery processes following ultrafast demagnetization [27,28], appearing as transient topological objects which mediate interactions between skyrmions, and are thus essential for a full understanding of such processes. It is interesting to note the formation of topological defects following phase transitions seems to be a wider phenomenon, previously

\footnotetext{
*slepadatu@uclan.ac.uk
}

discussed in relation to cosmological phase transitions [29], generation of defects in superfluid ${ }^{4} \mathrm{He}$ [30] and liquid crystals [31], and thus the study of such processes could have wider implications beyond topological magnetic solitons.

$$
Q_{i}=\frac{1}{4 \pi} \int_{A} \mathbf{m}_{i} \cdot\left(\frac{\partial \mathbf{m}_{i}}{\partial x} \times \frac{\partial \mathbf{m}_{i}}{\partial y}\right) d x d y, \quad(i=A, B) .
$$

Studies of ultrafast magnetization dynamics have revealed the possibility of all-optical switching (AOS) of magnetization, initially in ferrimagnetic GdFeCo [32], where a helicity dependence (HD-AOS) of magnetization switching for a train of circularly polarized ultrafast laser pulses has been observed. Explanations of this helicity dependence have been given in terms of the effective field created due to the inverse Faraday effect [32,33], as well as magnetic circular dichroism [34]. On the other hand, it has also been shown heating alone due to the laser pulse can also result in deterministic switching of magnetization without a helicity dependence $[35,36]$, where the sample passes through a transient ferromagneticlike state. HD-AOS has now been observed in a wide range of ferromagnetic systems, alloys, and multilayers $[37,38]$, where deterministic switching of magnetization was observed for a train of circularly polarized laser pulses. Additionally, ultrafast magnetization switching has also been demonstrated using electronic heat currents [39,40], and deterministic switching using single-shot linearly polarized laser pulses was demonstrated in a synthetic ferrimagnetic racetrack [41]. The possibility of creating skyrmions in magnetic thin films was also investigated experimentally. Using ultrashort laser pulses with varying fluence, Bloch skyrmions, stabilized mainly by dipole-dipole interactions, were created in ferrimagnetic TbFeCo $[42,43]$. Skyrmions have also been experimentally created using laser heat pulses in ferromagnetic FeGe, where Bloch skyrmions are stabilized by the bulk DMI [44]. In another experiment on $\mathrm{CoFeB} / \mathrm{Ta}$ multilayers, 
where Néel skyrmions are stabilized by interfacial DMI, illumination with single-shot ultrafast laser pulses resulted in formation of skyrmion clusters, with skyrmion density dependent on the laser pulse fluence [45]. Importantly, there was no difference observed in skyrmion formation between laser pulses with different helicities, and the skyrmion creation mechanism was attributed to heating.

Here we investigate in detail the dynamical skyrmion creation process following ultrafast demagnetization in antiferromagnetic (AFM) and ferromagnetic (FM) thin films, and show transient DW skyrmions are formed after recovery of magnetization order. While deterministic switching of magnetization is possible, we discuss the question if a single Néel skyrmion can be deterministically created using ultrafast laser pulses. We show that for both linearly and circularly polarized laser pulses, the possibility of a skyrmion with DW skyrmion pair self-annihilation is a barrier to deterministic creation of a single Néel skyrmion. Using thousands of skyrmion creation events we further analyze the statistical properties of skyrmion creation as a function of laser pulse properties, both in AFM and FM cases, showing the final states obey a Poisson counting distribution.

\section{DYNAMICAL SKYRMION CREATION PROCESS}

Theoretically, skyrmion creation using ultrafast laser pulses was investigated for Néel skyrmions using vortex laser pulses, where skyrmions are created due to the effective magnetic field $[46,47]$, while creation of skyrmions in antiferromagnetic insulators due to the inverse Faraday effect has also been studied [48]. On the other hand, creation of Bloch skyrmions and antiskyrmions has been investigated both in magnets with predominant dipolar interactions, as well as chiral magnets [49], where Langevin dynamics were taken into account during a rectangular heat pulse. Here we investigate the creation of Néel skyrmions both in AFM and FM materials, taking into account stochasticity both during and after a heat pulse. First we concentrate on the AFM case, then compare the skyrmion creation mechanism to that in the FM case.

The geometry studied is shown in Fig. 1(a). Here we use a $1-\mu \mathrm{m}^{2}, 2$-nm-thick magnetic thin film on top of an 8-nm-thick Pt layer on a $\mathrm{SiO}_{2}$ substrate, $40 \mathrm{~nm}$ thick. Further details and material parameters are given in Appendix B. The heat transport is solved using the twotemperature model [50-52], with continuity of heat flux and temperature across the interfaces, and Robin boundary conditions on the exposed surfaces of the magnetic layer and substrate [53]. The electron temperature is used in a twosublattice stochastic Landau-Lifshitz-Bloch model, which includes contributions from dipole-dipole interactions, direct and interfacial DMI exchange, antiferromagnetic exchange including both homogeneous and nonhomogeneous inter-lattice contributions, uniaxial anisotropy, and applied field. Full details are given in Appendix A and Ref. [54]. The effect of a linearly polarized laser pulse is included as a Gaussian heat source in the electron temperature heat equation as

$$
S=P_{0} \exp \left(\frac{-\left|\mathbf{r}-\mathbf{r}_{0}\right|}{d^{2} / 4 \ln (2)}\right) \exp \left(\frac{-\left(t-t_{0}\right)^{2}}{t_{R}^{2} / 4 \ln (2)}\right) \quad\left(W / m^{3}\right),
$$
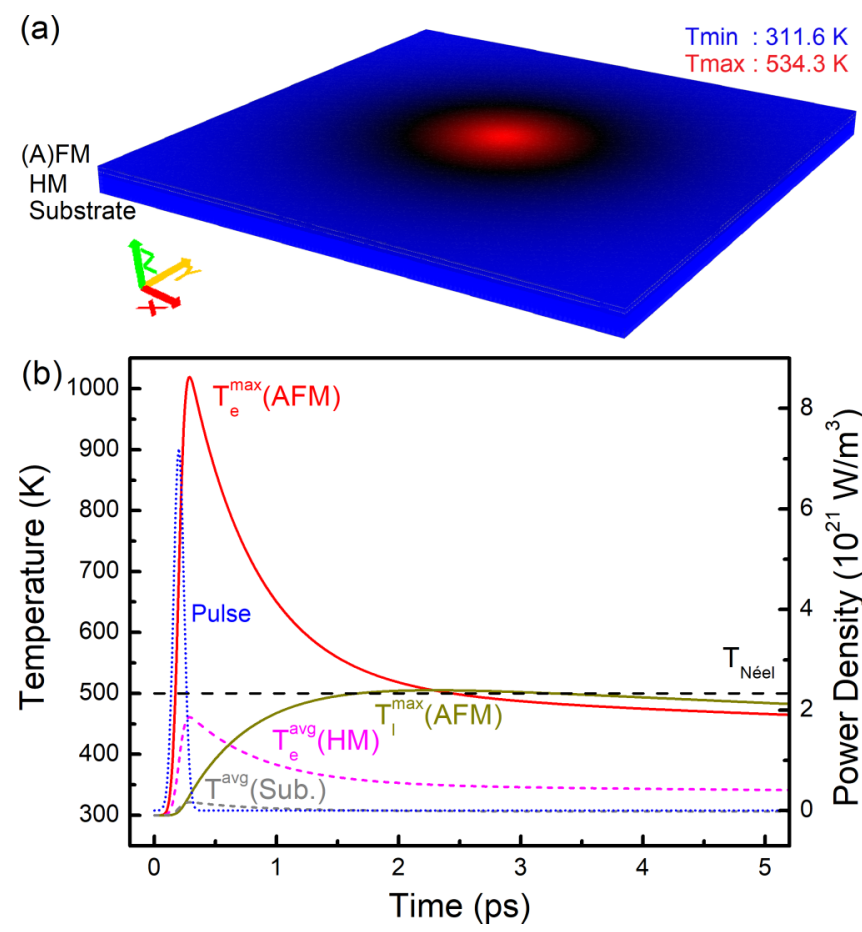

FIG. 1. (a) Temperature during a Gaussian profile laser pulse in an (anti)ferromagnetic $(2 \mathrm{~nm}) /$ heavy metal $(8 \mathrm{~nm}) /$ substrate $(40 \mathrm{~nm})$ structure. (b) Typical ultrafast laser pulse and temperature time dependence in the layers: magnetic layer maximum electron and lattice temperatures, heavy metal layer average electron temperature, and substrate average temperature.

where $d$ and $t_{R}$ are full width at half maximum (FWHM) values. Initially we fix $d$ to $800 \mathrm{~nm}$ and $t_{R}$ to $100 \mathrm{fs}$--later we also consider narrower and longer pulses, and also circularly polarized laser pulses by inclusion of the associated magnetooptical field. Curves for the electron and lattice temperatures in the different layers are shown in Fig. 1(b). Due to the small heat capacity of the electron bath, the maximum electron temperature rises very rapidly during the heat pulse, exceeding the Néel temperature for up to a few picoseconds, before converging towards the slower-changing lattice temperature, and eventually back towards room temperature on a longer timescale lasting beyond $1 \mathrm{~ns}$.

A typical skyrmion creation process is shown in Fig. 2 for a single high-power linearly polarized laser pulse $(7 \times$ $10^{21} \mathrm{~W} / \mathrm{m}^{3}$ ), plotting the $z$ component of magnetization on sublattice $A$; typically we distinguish several stages in the skyrmion creation process. The timing of these stages discussed below depends on the laser power, and even for the same power there is some variation and uncertainty in describing exactly where a stage starts and ends, although we can more precisely define them by analyzing the time dependence of the topological charge. The first stage is demagnetization during the applied laser pulse, where the temperature rises rapidly and phase transition to the paramagnetic state occurs for $T>T_{\text {Néel }}$; at the end of the laser pulse $A_{\mathrm{PM}}$ is reached, which is the maximum area around the central spot with $T>$ $T_{\text {Néel }}$. From this point on the temperature starts to decrease and, as it drops below $T_{\text {Néel }}$, areas with reversed magnetization direction emerge, first at the outer boundary of $A_{\mathrm{PM}}$, then 


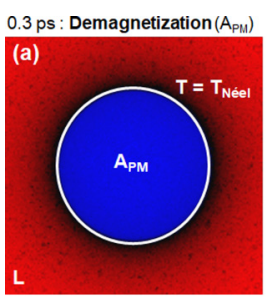

4-10ps: Skyrmioncreation

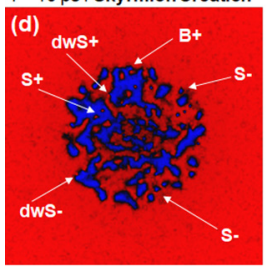

$50-700$ ps : Pair Annihilation
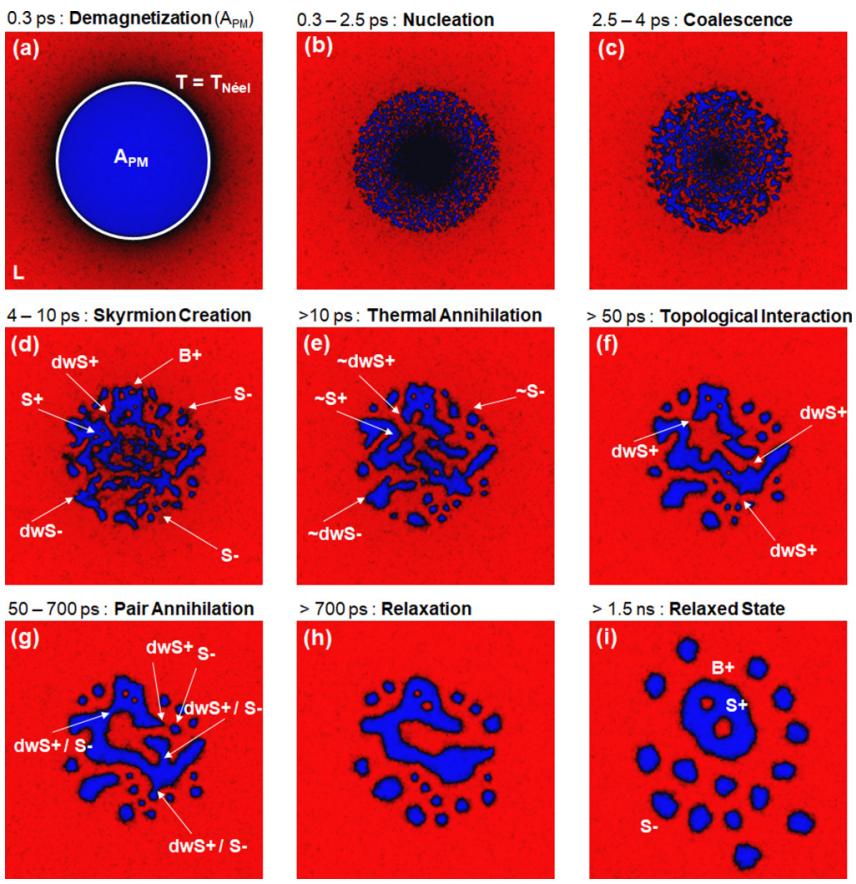

$>1.5$ ns: Relaxed State

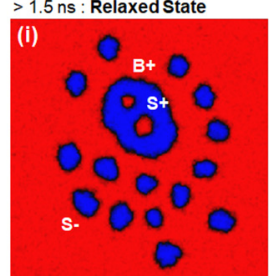

FIG. 2. Exemplification of the different stages observed after a linearly polarized $(\mathrm{L})$ high-power $\left(7 \times 10^{21} \mathrm{~W} / \mathrm{m}^{3}\right)$ laser pulse, showing the $z$-magnetization component on sublattice A. (a) Demagnetization, showing the paramagnetic area $\left(A_{\mathrm{PM}}\right)$. (b) Nucleation of reversed domains as the temperature drops below $T_{\text {Néel }}$. (c) Coalescence of nucleated domains. (d) Skyrmion creation due to DMI, showing examples of $\mathrm{S} \pm, \mathrm{dwS} \pm$, and $\mathrm{B}+$. (e) Thermal annihilations of skyrmions. (f) Topological interactions (annihilation and repulsion) become important over a longer timescale. (g) Pair annihilations, showing dwS $+/ \mathrm{S}-$ annihilations. (h) Relaxation towards the final state starts after a stable topological charge is reached. (i) Relaxed state with minimal skyrmion distortions and larger spacings due to repulsive topological interactions reached on a timescale of ns.

moving inwards towards the center as the area with $T>T_{\text {Néel }}$ contracts. The nucleation and growth model, where reversed domains are nucleated through thermal activation over an energy barrier following an Arrhenius law [55,56], cannot be used to explain the nucleation stage observed here. Such processes occur on longer timescales of 10-100 ps and longer, while the ultrafast nucleation stage occurs on a timescale of $\sim 1$ ps. The nucleation stage is due to the much faster longitudinal relaxation process $[57,58]$, and requires quenching of net magnetization. As the temperature cools below the phase transition temperature, the nucleons of reversed magnetization formed in the quenched state give rise to domains with reversed magnetization. The next stage consists of coalescence of nucleated domains where, due to the high density of nucleation centers, the nucleated domains very rapidly coalesce into larger domains. Such localization and coalescence processes have also been identified recently in ferrimagnetic alloys [59]. Since the temperature is not uniform, as for the nucleation stage, coalescence first starts at the outer boundaries of $A_{\mathrm{PM}}$, proceeding towards the center as seen in Fig. 2(c). As larger domains emerge, topologically protected structures become
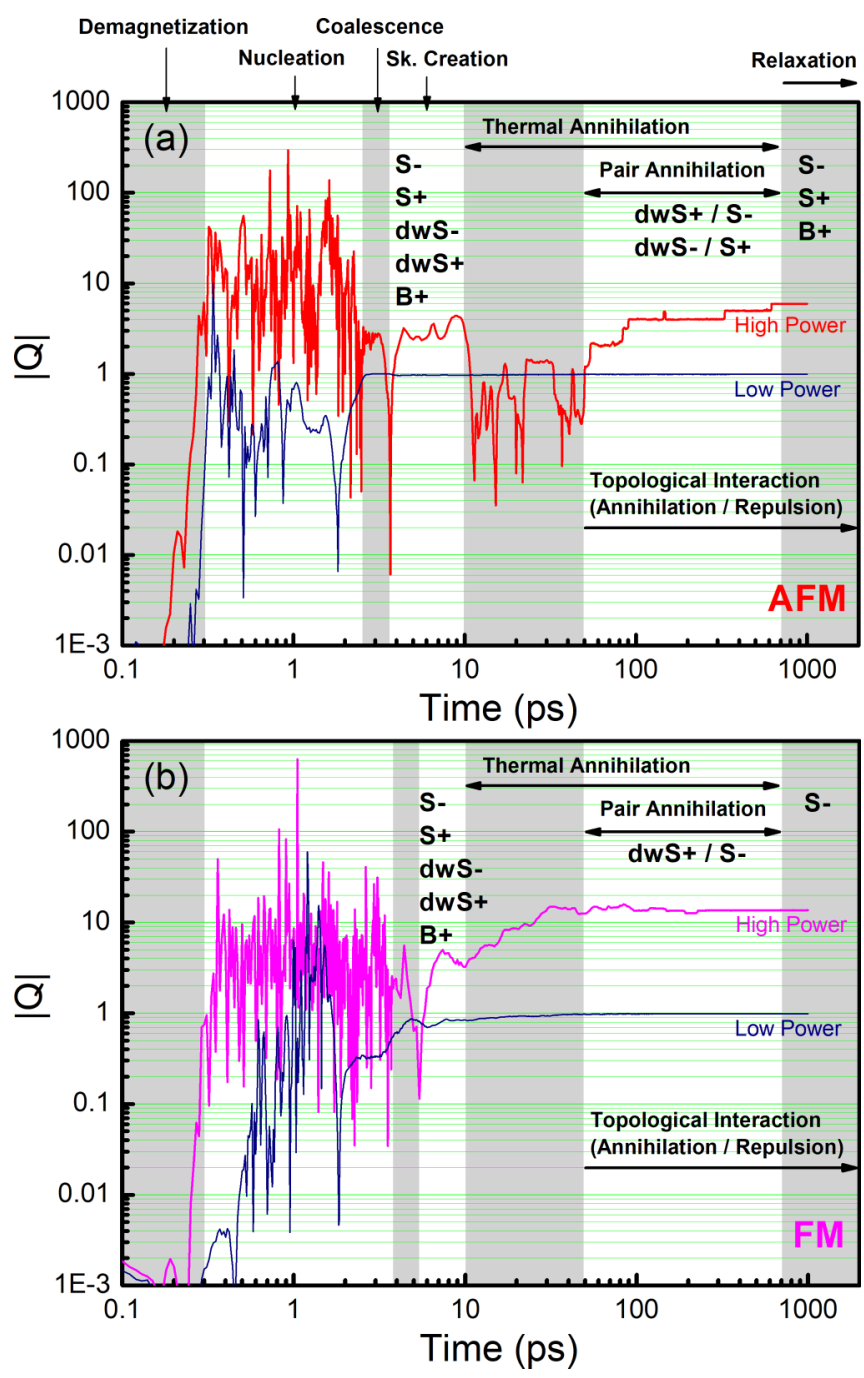

FIG. 3. $|Q|$ plotted as a function of time for both (a) AFM sublattice A, and (b) FM at high $\left(7 \times 10^{21} \mathrm{~W} / \mathrm{m}^{3}\right)$ and low $(3 \times$ $10^{21} \mathrm{~W} / \mathrm{m}^{3}$ ) laser powers with linear polarization. During the nucleation process the $|Q|$ value changes randomly due to thermal fluctuations and highly disordered magnetization, and is not a welldefined measure. The nucleated domains coalesce, typically resulting in a sharp drop in $|Q|$ towards the real topological charge at this stage: zero. Under the action of DMI, topological objects start to emerge as Néel domain walls are formed, resulting in a sharp increase in $|Q|$ which signals the start of the skyrmion creation stage. Many of the topological objects are gradually destroyed due to thermal activation, resulting in fluctuations in $|Q|$. On a longer timescale topological interactions become important, including pair annihilations and repulsive forces. Finally, a stable topological charge is reached, with relaxation continuing as a result of energy minimization, and in particular repulsive topological interactions. The same stages can be observed at low powers, but the timescales are shorter.

gradually distinguishable, formed from the disordered domain wall magnetization under the effect of DMI. This is reflected by a rapid increase in the topological charge as seen in Fig. 3(a) for the skyrmion creation stage.

Here $|Q|$ is plotted as a function of time, computed using Eq. (1), showing features for typical skyrmion creation processes at high $\left(7 \times 10^{21} \mathrm{~W} / \mathrm{m}^{3}\right)$ and low $\left(3 \times 10^{21} \mathrm{~W} / \mathrm{m}^{3}\right)$ 
TABLE I. List of observed skyrmions. The skyrmions are characterized on sublattice A for the AFM case, showing the topological charge $Q(A)$, and indicating the topological objects found in the relaxed state for AFM and FM cases.

\begin{tabular}{llcc}
\hline \hline Symbol & \multicolumn{1}{c}{ Name } & Q(A) & Relaxed state \\
\hline $\mathrm{S}-$ & Skyrmion & -1 & AFM, FM \\
$\mathrm{S}+$ & Skyrmion & +1 & AFM \\
$\mathrm{dwS}-$ & Domain wall skyrmion & -1 & None \\
$\mathrm{dwS}+$ & Domain wall skyrmion & +1 & None \\
$\mathrm{B}-(|Q|)$ & Skyrmion bag (skyrmionium) & $\leqslant 0(0)$ & AFM (probability $\left.<10^{-3}\right)$ \\
$\mathrm{B}+(|Q|)$ & Skyrmion bag (skyrmionium) & AFM \\
\hline \hline
\end{tabular}

laser powers. During the nucleation stage the $Q$ value is ill defined due to the high degree of magnetization disorder. As the coalescence stage starts, the noise in the computed $Q$ value very rapidly settles, and a sharp drop towards zero is observed in the vast majority of cases, both for AFM and FM, and both at high and low powers. After this drop, typically a sharp rise towards a well-defined value of $|Q|$ signifies the start of the skyrmion creation stage as noted above. At the end of the skyrmion creation process the reversed domains are separated by Néel domain walls, although very significant distortions are still present in the skyrmions at this stage as seen in Fig. 2(d). During the skyrmion creation stage, for both AFM and FM cases, the most common type of skyrmion formed is the $\mathrm{S}$ - skyrmion--for a full list see Table I. S+ skyrmions are also formed, although these are much rarer since they require nucleation of a domain within another reversed domain. When they are created however, they form skyrmion bags, $\mathrm{B}+$. It is also possible for a B - skyrmion bag to be formed; however, this is extremely rare since they require an $\mathrm{S}+$ within an $\mathrm{S}-$, within an $\mathrm{S}+$ skyrmion-in 3000 events this was observed only twice, and in both cases a B-(0) skyrmionium was formed.

Another type of topological object formed, which is ubiquitous in both AFM and FM cases, is the DW skyrmion [22-25] with topological charge of \pm 1 , i.e., dwS \pm ; examples are indicated in Fig. 2(d). The domain wall skyrmion is a $360^{\circ}$ rotation of the in-plane magnetization components transverse to an out-of-plane Néel domain wall, stabilized by the DMI and topologically protected. Fig. 4 shows examples of dwS+ relaxed at $0 \mathrm{~K}$, attached to an antiferromagnetic skyrmionium in Fig. 4(a), and to a simple ferromagnetic Néel domain wall in Fig. 4(b). While a large population of $\mathrm{dwS} \pm$ are created during the skyrmion creation stage, these objects never survive in the relaxed state owing to a combination of rapid thermally activated collapse, as well as pair annihilations with $\mathrm{S} \pm$, which could explain why such topological structures have not yet been observed in experiments. Skyrmions and DW skyrmions with opposite topological charge experience an attractive topological interaction, mainly owing to spatial gradients in the DMI energy, while dipole-dipole interactions also play a role in the FM case at larger separations [60]. When sufficiently close, this results in a pair annihilation with no net change in the topological charge. Examples of such annihilations, in particular $\mathrm{dwS}+/ \mathrm{S}-$ annihilations, have been identified in Figs. 2(f) and 2(g)-as result of a pair annihilation, the isolated $\mathrm{S}-$ skyrmion is effectively absorbed by the $\mathrm{S}-$ skyrmion with the $\mathrm{dwS}+$ attached, which results in the unwinding of the dwS + structure. On the other hand, skyrmions and DW skyrmions with the same topological charge experience a repulsive topological interaction, similar to that observed between skyrmions. Thus in Fig. 4(a) the $\mathrm{B}+(0)-\mathrm{dwS}+$ pair is stable owing to the topological repulsion between the dwS + and the $\mathrm{S}+$ skyrmion inside the skyrmionium. If on the other hand a single $\mathrm{dwS}+$ is attached to an $\mathrm{S}-$ skyrmion, the pair is not stable even at $0 \mathrm{~K}$--owing to the topological attraction such a pair rapidly contracts, resulting in self-annihilation where both the $\mathrm{S}-$ and $\mathrm{dwS}+$ are absorbed into the background state. However, if multiple dwS+ objects are attached to a single $\mathrm{S}-$, the structure becomes stable at $0 \mathrm{~K}$ due to the repulsive interaction between the $\mathrm{dwS}+$ objects, which prevents collapse of the $\mathrm{S}-$ skyrmion.

After creation, many topological objects are thermally annihilated as exemplified in Fig. 2(e), including skyrmions far
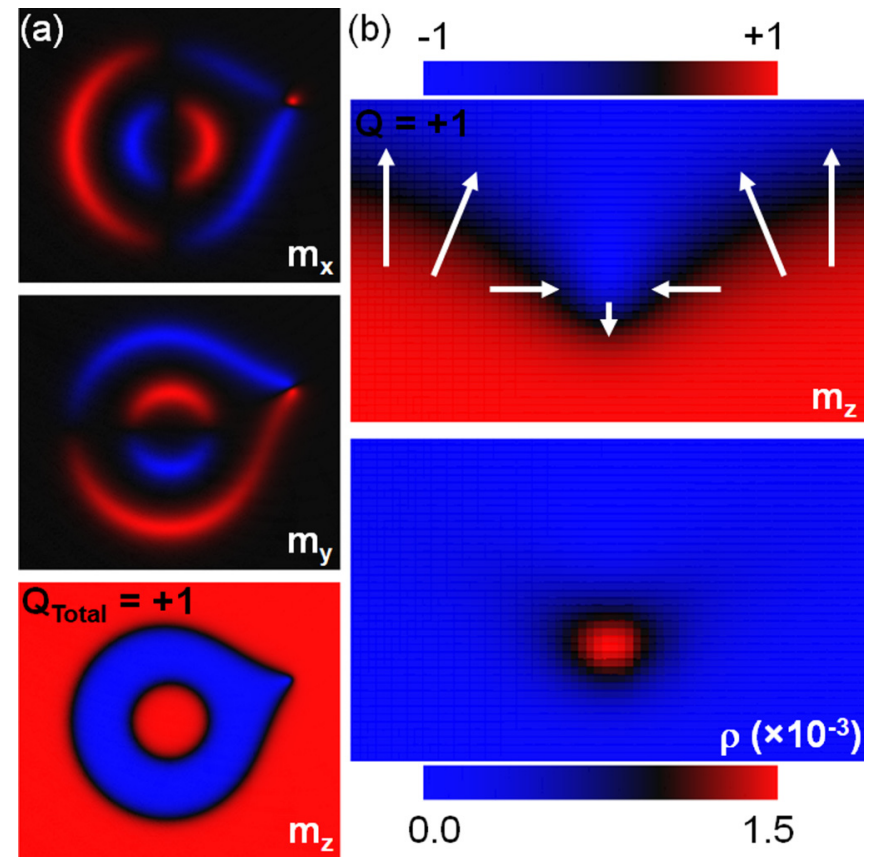

FIG. 4. (a) Stable antiferromagnetic skyrmionium-DW skyrmion pair $[\mathrm{B}+(0)-\mathrm{dwS}+]$ with total topological charge of +1 on sublattice A, showing the individual magnetization vector components. (b) Ferromagnetic DW skyrmion showing the $z$ component, with overlaid sketch of the in-plane components of magnetization. The panel below shows the corresponding topological charge density, $\rho$. All magnetization configurations are relaxed at $T=0 \mathrm{~K}$. 

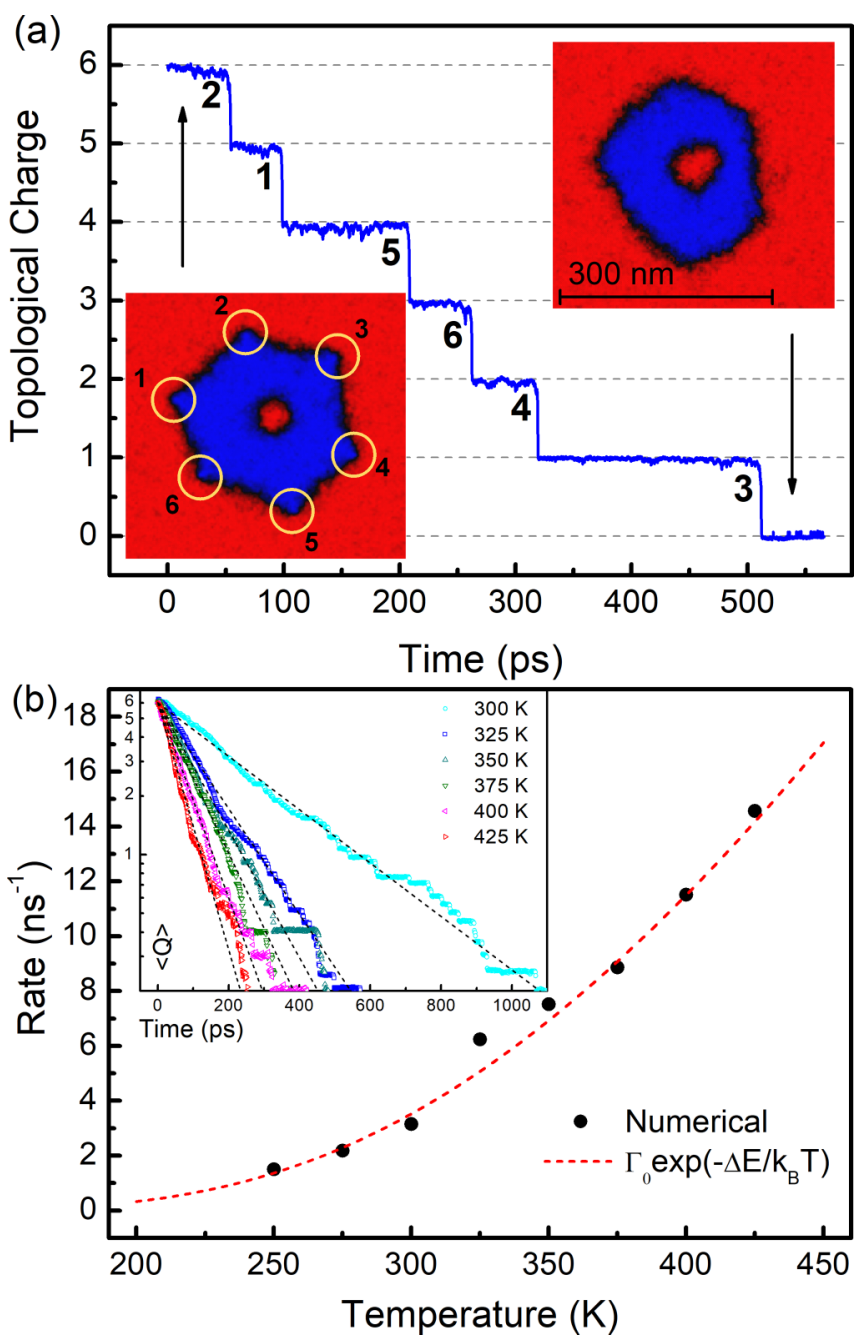

FIG. 5. Thermal decay of an AFM skyrmionium-DW skyrmion complex with total topological charge of +6 is shown, for (a) single decay at $325 \mathrm{~K}$, and (b) averaged decay for a range of temperatures. The decay is described by an exponential process with a decay rate constant following an Arrhenius law with energy barrier $\Delta E=2 \times$ $10^{-20} \mathrm{~J}$ and attempt frequency $\Gamma_{0}=4 \times 10^{11} \mathrm{~s}^{-1}$.

away from equilibrium, while $\mathrm{dwS} \pm$ objects are inherently susceptible to rapid thermally activated collapse as we discuss below. This results in fluctuations in the computed value of $|Q|$ as shown in Fig. 3, both for AFM and FM: due to a preponderance of $\mathrm{S}-$, collapse of $\mathrm{dwS}+$ and $\mathrm{S}+$ increases the $|Q|$ value, while collapse of dwS - and $\mathrm{S}-$ decreases it. Finally, as all the pair and thermal annihilations of unstable objects have completed, the final $Q$ value is reached; from this point the skyrmions continue to relax-Fig. 2(h)--expanding beyond $A_{\mathrm{PM}}$ under the action of repulsive topological interactions, eventually reaching a relaxed state on a timescale of nanoseconds. It has been shown that skyrmions decay through thermal activation as described by an Arrhenius law, both for Néel FM skyrmions [61,62], Bloch FM skyrmions [63], and AFM skyrmions [64], with experimental verification available in a chiral magnet [65]. Here we show DW skyrmions also decay through thermal activation following an Arrhenius law. Figure 5 shows the thermal decay of AFM dwS+, where a skyrmionium with six attached $\mathrm{dwS}+$ is isolated, and a typical thermal decay is shown in Fig. 5(a) at $325 \mathrm{~K}$.

While the DW skyrmions are also topologically protected, they can collapse through thermal activation at a faster rate compared to FM or AFM skyrmions, both of which have lifetimes on timescales of ns or longer at room temperature, depending on material parameters. The thermally activated collapse of skyrmions arises through a gradual contraction in the diameter, until flipping of the core magnetization results in loss of the topological structure [61]; another possibility is by nucleation of a singularity resembling a hedgehog Bloch point [62]. On the other hand, the thermal collapse of a DW skyrmion is much simpler since the topological charge density is highly localized, as seen in Fig. 4(b). Thus we only require the central in-plane components to flip, which is driven by thermal activation over an energy barrier. To see this, we compute the average topological charge over 30 decays of the skyrmionium-DW skyrmion complex for each temperature, with results shown in Fig. 5(b). The thermal decay is exponential with a single decay rate dependent on temperature, confirming the thermal collapse of the $6 \mathrm{dwS}+$ is independent of the number of $\mathrm{dwS}+$ attached to the skyrmionium. The decay rate follows an Arrhenius law as a function of temperature, $\Gamma=\Gamma_{0} \exp \left(-\Delta E / k_{\mathrm{B}} T\right)$, where we obtain the attempt frequency $\Gamma_{0}=4 \times 10^{11} \mathrm{~s}^{-1}$ and the energy barrier $\Delta E=$ $2 \times 10^{-20} \mathrm{~J}$. This energy barrier is comparable to that obtained for FM skyrmions [61]; however, the attempt frequency is much higher, resulting in rapid thermal decay on timescales of ps at room temperature. This is in agreement with results obtained in Ref. [64] for AFM materials, where it was shown that large attempt frequencies result in stability timescales of milliseconds for temperatures in the range $50-65 \mathrm{~K}$ for reasonable material parameters, which is comparable to the stability of AFM DW skyrmions obtained here in the same temperature range. It should be noted $\mathrm{dwS}-$ are significantly rarer than $\mathrm{dwS}+$, because in a $\mathrm{dwS}--\mathrm{S}-$ pair the added repulsive topological interaction, which favors flipping of the $\mathrm{dw} S$ center, causes the $\mathrm{dwS}-$ object to collapse much quicker than a dwS+.

\section{SINGLE NÉEL SKYRMION CREATION}

Whilst a large population of skyrmions is typically created at high powers, it is possible to create a single skyrmion at low powers, as shown in Fig. 3. It is important to note however that this is not a deterministic process in the cases studied here, both for AFM and FM: Once a skyrmion is created there is a distinct probability of thermally activated collapse after creation when the skyrmion is still far away from equilibrium, which occurs on timescales of up to $100 \mathrm{ps}$. This is in contrast to a previous work on Bloch skyrmions [49], which investigated conditions under which a single skyrmion can be created with $100 \%$ efficiency as a result of an ultrafast heat pulse. There the temperature was set to zero after the heat pulse, thus removing the thermal activation process which can lead to skyrmion collapse for the cases studied here. As shown in Fig. 1 the temperature decays on a much longer timescale compared to the ultrafast heat pulse, with the temperature changing slowly after the initial temperature spike. The stochasticity of magnetization dynamics must be taken 

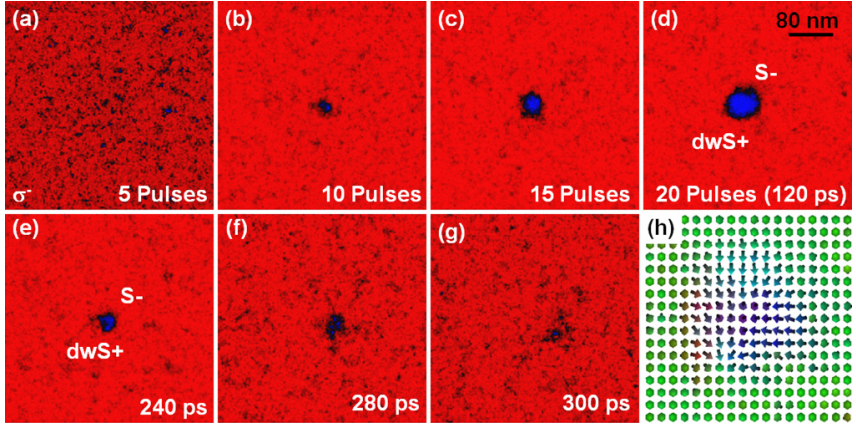

FIG. 6. Skyrmion self-annihilation with a domain wall skyrmion. A train of 20 circularly polarized laser pulses, heating the material close to, but below the Curie temperature, are applied with a negative helicity $\left(\sigma^{-}\right)$. (a)-(d) Magnetization state, showing the $z$ component, after the indicated number of pulses. After the last pulse an S-$\mathrm{dwS}+$ pair is visible, with a close-up magnetization configuration shown in (h). (e)-(g) Collapse and self-annihilation of S--dwS+ pair due to topological attraction.

into account during this stage, which can result in a range of possible outcomes for the final relaxed state. Experimental results on Néel skyrmion formation after an ultrafast heat pulse have also shown random skyrmion nucleation at low laser fluences [45]; however, the formation of Bloch skyrmion bubbles with $100 \%$ efficiency was reported in experiments on a dipolar magnet [42]. Another possibility, intrinsic to the Néel skyrmions studied here, is for a $\mathrm{S}--\mathrm{dwS}+$ pair to be nucleated which results in collapse of the skyrmion and finally self-annihilation even in the absence of stochasticity.

The remaining question is whether a single Néel skyrmion can be created deterministically using a train of laser pulses, instead of a single-shot pulse, and additionally using circularly polarized light where HD-AOS arises. It has been shown experimentally that no helicity dependence exists in a FM material with interfacial DMI [45] for single-shot ultrafast laser pulses. If the main mechanism giving rise to a helicity dependence is the inverse Faraday effect, where a perpendicular magneto-optical field is present during the laser pulse, this observation is not surprising since precessional magnetization processes require significantly longer time to respond to the magnetic field than available in a single sub-ps laser pulse. Instead, the accumulated effect of a train of laser pulses is required to deterministically switch magnetization with a helicity dependence in FM materials [66-68]. Thus, while a large area can be switched deterministically using a train of laser pulses [37], it remains an open question whether a single Néel skyrmion can be deterministically created under appropriate conditions. It has already been shown the HDAOS reversed domain size needs to be larger than the laser spot size [69]. Here we show that even with a train of circularly polarized laser pulses which heat the material close to the phase transition temperature, the possibility of nucleating a $\mathrm{S}-$-dwS + pair or complex cannot be disentangled from the possibility of creating just one $\mathrm{S}-$. Due to the circular polarization of the laser pulse a strong perpendicular magnetooptical field is present, given by $H_{M O}=\sigma^{ \pm} H_{M O}^{0} f_{M O}(\mathbf{r}, t) \hat{\mathbf{z}}$ [33], where $f_{M O}$ has the spatial and temporal dependence given in Eq. (2), and $\sigma^{ \pm}= \pm 1$. The results in Fig. 6 are shown for the FM case, where we apply a sequence of 20 circularly polarized laser pulses at 6-ps intervals with negative helicity $\left(\sigma^{-}\right)$and strength of $10 \mathrm{MA} / \mathrm{m}$. An out-of-plane bias field of $100 \mathrm{kA} / \mathrm{m}$ is used, and we have checked the positive helicity does not result in switching of magnetization. Here $t_{R}=500 \mathrm{fs}$, and $d=100 \mathrm{~nm}$, which is slightly larger than the ideal skyrmion diameter of $80 \mathrm{~nm}$. Throughout the pulse sequence in Fig. 6 the temperature does not exceed the Curie temperature. The cumulative effect of laser pulses is to gradually reduce the magnetization in the central spot due to the higher temperature, and eventually a small reversed domain is nucleated under the strong magneto-optical field, Fig. 6(b). As further pulses are applied this reversed domain grows until a maximum size is reached, Figs. 6(c) and 6(d). While this results in a single $\mathrm{S}$ - skyrmion in some cases, due to the large degree of disorder at the skyrmion boundary there is a distinct probability of nucleating one or more $\mathrm{dwS}$, as is the case in Fig. 6(d) - -a close-up of the magnetization structure is shown in Fig. 6(h), clearly identifying an $\mathrm{S}--\mathrm{dw} \mathrm{S}+$ pair. As a result of the topological attraction between the $\mathrm{S}-$ and dwS+, the skyrmion quickly collapses-Figs. 6(e) and 6(f)--and is annihilated, Fig. $6(\mathrm{~g})$. Finally, there is the possibility that creating a large enough skyrmion avoids a self-annihilation collapse if the DW skyrmion lifetime is significantly shorter than the skyrmion collapse time. We have also investigated this; however, typically for a large skyrmion the border tends to have significant distortions after ultrafast laser pulses, with many $\mathrm{dwS} \pm$ present, which rather than cause a complete collapse typically result in the skyrmion splitting into multiple skyrmions; material defects and multilayers will further complicate this process, however this is beyond the scope of this work.

\section{STATISTICAL PROPERTIES}

We have already remarked on the differences between skyrmion creation at high and low laser powers. Here we systematically study the statistical properties of skyrmion creation as a function of heat pulse properties, first for the AFM case, and in the next section we investigate the differences for the FM case. For this, a laser pulse with linear polarization is applied, and when a stable topological charge is reached the number of skyrmions created is computed. For each heat pulse setting this process is repeated up to 50 times.

The results are shown in Fig. 7. Here we use two values of pulse width and duration, namely $d=400 \mathrm{~nm}, 800 \mathrm{~nm}$, and $t_{R}=100 \mathrm{fs}, 500 \mathrm{fs}$, and vary the pulse power. The variation of $A_{\mathrm{PM}}$ with maximum temperature reached for the different pulse settings is shown in the inset to Fig. 7(b), and Fig. 7(a) shows the mean number of skyrmions created as a function of $A_{\mathrm{PM}}$. While there is a significant statistical uncertainty in obtaining the mean skyrmion creation rates due to the limited number of events included, the rates for $\mathrm{S}-, \mathrm{S}+$, and $\mathrm{B}+$ follow the same trend lines, respectively, when plotted as a function of $A_{\mathrm{PM}}$, even though the pulse characteristics are otherwise very different. This can be understood from the dynamical skyrmion creation process, in particular the nucleation stage--see Fig. 3(b). Since the average number of nucleated domains is dependent on $A_{\mathrm{PM}}$, then so is the final number of stable skyrmions. The maximum temperature 


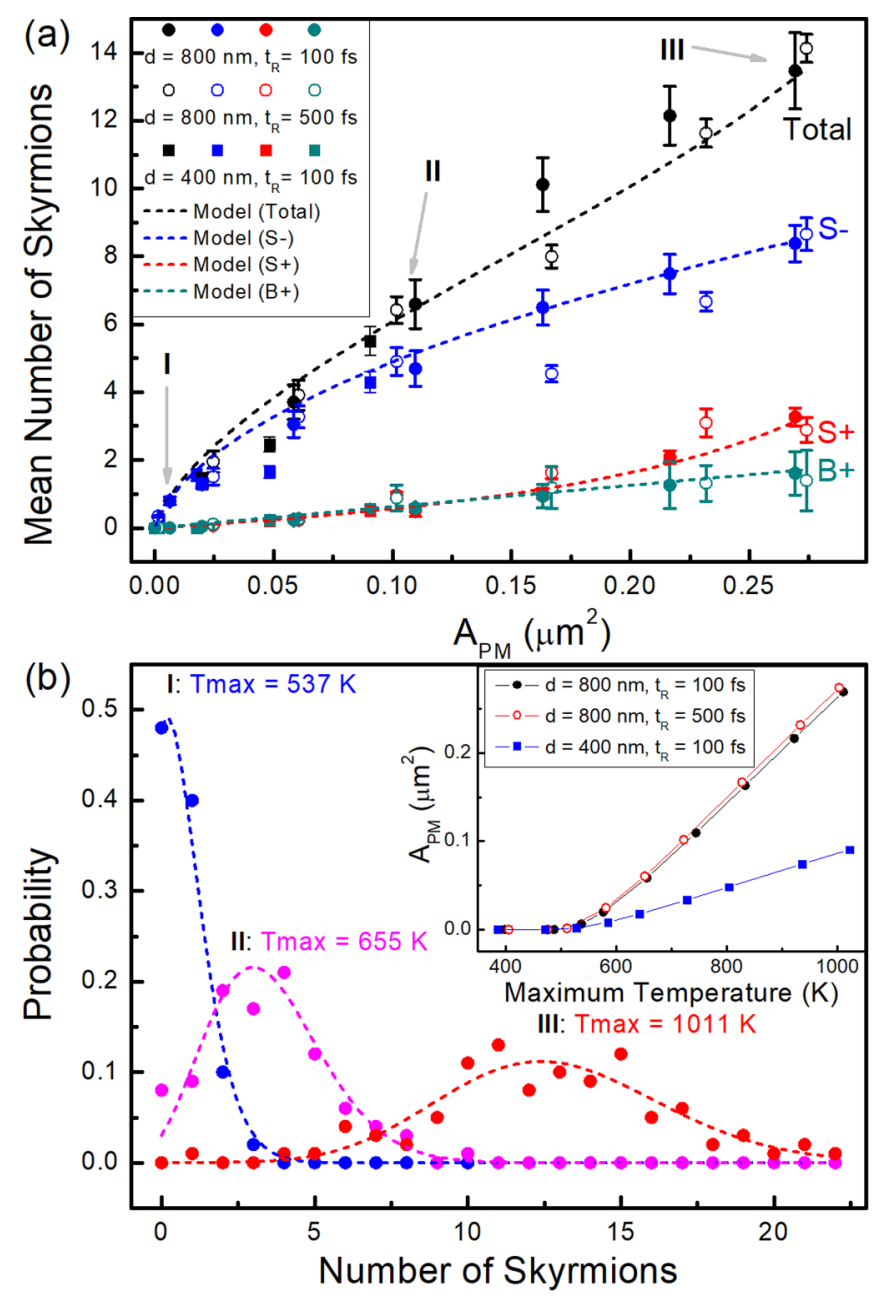

FIG. 7. (a) Mean number of skyrmions created is shown as a function of $A_{\mathrm{PM}}$ for FWHM pulse values of $d=400 \mathrm{~nm}, 800 \mathrm{~nm}$, and $t_{R}=100 \mathrm{fs}, 500 \mathrm{fs}$. The different types of stable topological objects formed are shown, namely $\mathrm{S}-, \mathrm{S}+$, and $\mathrm{B}+$. The dashed lines are obtained from the model in Eq. (3). (b) Skyrmion creation probability distributions are shown for three selected pulse strengths, with fitted Poisson distributions-the values in (a) are the fitted mean rates together with fitting uncertainties, obtained from 50 repetitions for each pulse setting. The inset shows $A_{\mathrm{PM}}$ as a function of maximum temperature reached for the different pulses used.

reached does not have a noticeable effect on the skyrmion creation rates for the same $A_{\mathrm{PM}}$, serving only to reach a quenched magnetization state. Another important observation is the number of skyrmions created follows a Poisson distribution, i.e., $P(n)=\lambda^{n} e^{-\lambda} / n$ !, where $n$ is the number of skyrmions created and $\lambda$ is the mean skyrmion creation rate. Examples are shown in Fig. 7(b) for three different pulse powers-the mean skyrmion creation rate is obtained from a Poisson distribution fit.

$$
N_{S \pm}^{\mathrm{AFM}}=\frac{A_{\mathrm{PM}}}{A_{S \pm}^{\mathrm{AFM}}}\left[1+r_{ \pm}\left(N_{S \pm}^{\mathrm{AFM}}-1\right)\right]^{ \pm 1} .
$$

Next, we develop a simple phenomenological model to describe the variation in mean number of $\mathrm{S}-, \mathrm{S}+$, and $\mathrm{B}+$ objects created as a function of $A_{\mathrm{PM}}$. As $A_{\mathrm{PM}}$ increases, the number of more complex topological objects created, $\mathrm{B}+$, increases, which mirrors the experimental observations for Bloch skyrmions [42]. The increase in mean number of $\mathrm{S}-$ skyrmions is not linear, however, but slows down as $A_{\mathrm{PM}}$ increases, i.e., the density of $\mathrm{S}-$ decreases with $A_{\mathrm{PM}}$. As the number of $\mathrm{S}-$ skyrmions increases, due to the probability of nucleating $\mathrm{S}+$ skyrmions inside them, the number of $\mathrm{S}+$ also increases. The resultant $\mathrm{B}+$ objects tend to occupy a significantly larger area, which reduces the available area for $\mathrm{S}-$ skyrmions, and thus decreasing their density. On the other hand, the density of $\mathrm{S}+$ skyrmions increases slightly with $A_{\mathrm{PM}}$ due to the topological pressure experienced inside a skyrmion bag. The $\mathrm{B}+$ objects are constrained by topological repulsion from surrounding $\mathrm{S}-$ skyrmions. In turn this results in increased density of the contained $\mathrm{S}+$ skyrmions, as they experience a topological repulsion from neighboring $\mathrm{S}+$, as well as from the Néel border of the containing $\mathrm{B}+$; for example see Fig. 2(d). The model is shown in Eq. (3). Here $A_{S \pm}^{\mathrm{AFM}}$ are the paramagnetic areas required to create one $\mathrm{S} \pm$ skyrmion, respectively, on average, while $r_{+} \cong 0.3$ and $r_{-} \cong 0.4$ are fitting factors representing the increase, respectively, decrease, in $\mathrm{S} \pm$ skyrmion density with increasing $A_{\mathrm{PM}}$. The increase in $\mathrm{B}+$ with $A_{\mathrm{PM}}$ is assumed to be approximately linear. We have also repeated these calculations for varying DMI strength, with results shown in Ref. [70], where the same $r$ fitting factors are used. A reasonable agreement is obtained between the numerical results with statistical information extracted from 50 events for each set of simulation parameters, and the simple phenomenological model in Eq. (3).

In the case of FM, in addition to DMI, skyrmions also interact through the dipole-dipole interaction, which is an additional source of topological repulsion between skyrmions of same topological charge. Notwithstanding, there are strong similarities to the AFM case, some of which have been discussed in relation to Fig. 3. In terms of dynamical processes, the same stages seen in Fig. 2 are also observed for the FM case. The most important difference however, in the relaxed state only $\mathrm{S}-$ skyrmions are observed. Typical resultant relaxed stages are shown in Figs. 8(b) and 8(c): for the FM case the skyrmions relax into a hexagonal lattice as observed experimentally [45], while for the AFM case the final state is composed of a combination of $\mathrm{S}-, \mathrm{S}+$, and $\mathrm{B}+$ objects. As for AFM, dwS objects exhibit a quick thermal decay, and also participate in skyrmion-DW skyrmion pair annihilations. S+ skyrmions on the other hand are not stable, since the direction of the applied magnetic field favors stabilization of $\mathrm{S}-$ skyrmions only, and collapse on a timescale shorter than $1 \mathrm{~ns}$. For a broader discussion of dynamical properties of topological magnetic solitons in AFM materials the reader is referred to Ref. [16]. Thus while static properties of topological solitons are similar in FM and AFM cases, dynamical properties of solitons in AFM materials are strongly influenced by the exchange enhancement due to the Weiss exchange field. This results in much higher resonance frequencies up to the terahertz range, also influencing soliton velocities [16] and dynamical interactions between the two-dimensional magnetic topological solitons observed here; however, a detailed comparison between FM and AFM solitons is beyond the scope of this work. 

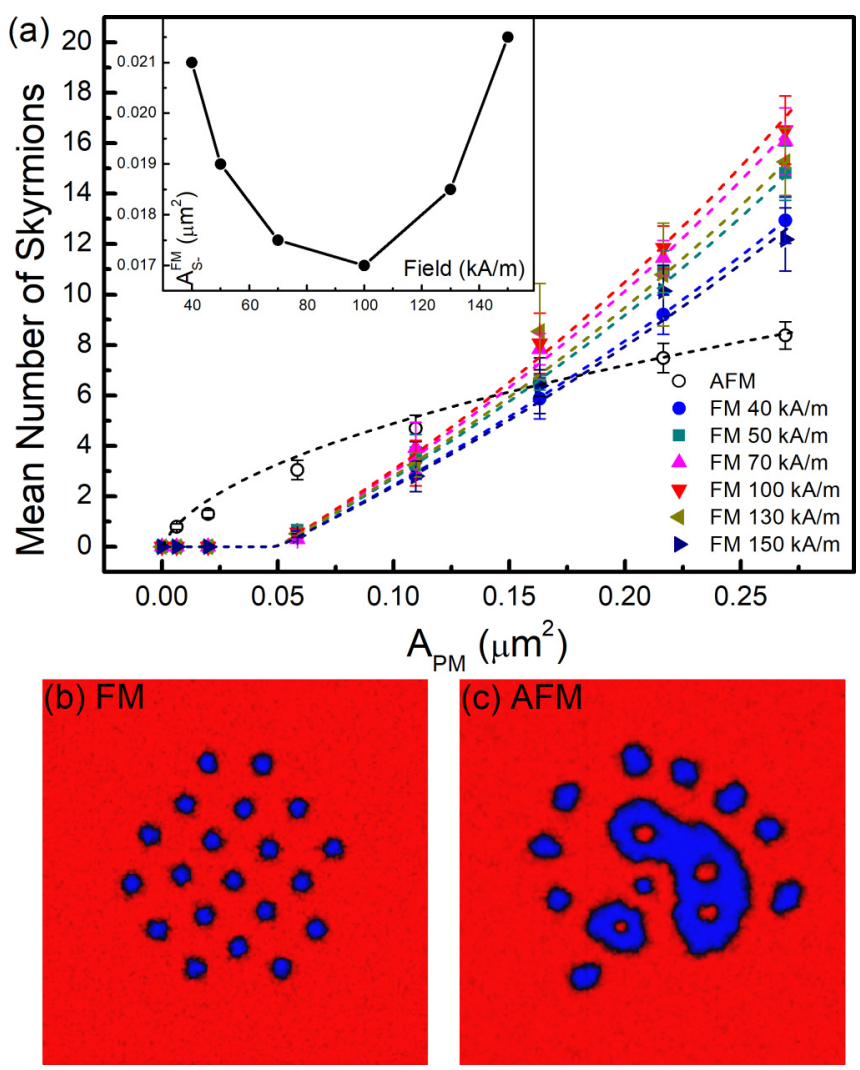

FIG. 8. (a) Mean number of $\mathrm{S}-$ skyrmions as a function of $A_{\mathrm{PM}}$, for out-of-plane field strength varying from $40 \mathrm{kA} / \mathrm{m}$ up to $150 \mathrm{kA} / \mathrm{m}$. The dashed lines are obtained from the model in Eq. (4). For comparison the $\mathrm{S}$ - antiferromagnetic skyrmion mean rates for $D=1 \mathrm{~mJ} / \mathrm{m}^{2}$ are also shown. The inset shows the area required to obtain one skyrmion on average as a function of applied field. (b) Typical created FM skyrmion cluster, and (c) typical created AFM skyrmion collection at high power $\left(7 \times 10^{21} \mathrm{~W} / \mathrm{m}^{3}\right)$.

In terms of statistical properties, the Poisson counting distribution is also obeyed by the resultant FM skyrmion states. The mean number of $\mathrm{S}-$ skyrmions is plotted in Fig. 8(a) as a function of $A_{\mathrm{PM}}$, and compared to the AFM case. In contrast to the AFM case, a threshold paramagnetic area $A_{0} \cong 0.05 \mu \mathrm{m}^{2}$ is required for any skyrmions to be formed. This is due to the paramagnetic susceptibility resulting in a net magnetic moment when a field is applied, and therefore a larger maximum temperature is required to reach the quenched magnetic state necessary for nucleation of reversed domains. We can recover the same physical picture by redefining the temperature value from which $A_{\mathrm{PM}}$ is calculated, or alternatively we can shift the zero point to $A_{0}$. As we have verified, the value of $A_{0}$ saturates quickly with fields greater than $1 \mathrm{kA} / \mathrm{m}$; however, the calculation of $A_{0}$ dependence on field and maximum temperature is beyond the scope of this study and is left for future work. As $A_{\mathrm{PM}}$ increases, the mean number of $\mathrm{S}-$ skyrmions is found to increase slightly faster than linear, which is a result of increasing skyrmion density with larger $A_{\mathrm{PM}}$. This is also in agreement with experimental observations [45], where long-distance dipole-dipole interactions result in compression of skyrmions at the center of $A_{\mathrm{PM}}$. Similar to the AFM case, the applicable phenomenological model is shown in Eq. (4), plotted in Fig. 8.

$$
N_{S-}^{\mathrm{FM}}=\frac{\left(A_{\mathrm{PM}}-A_{0}\right)}{\left(A_{S-}^{\mathrm{FM}}-A_{0}\right)}\left[1+r\left(N_{S-}^{\mathrm{FM}}-1\right)\right] .
$$

Here a much smaller $r$ factor of 0.02 is obtained, since the increase in density due to dipole-dipole interactions occurs over much larger distances compared to the DMI origin in the AFM case. The area required for single skyrmion creation, $A_{S-}^{\mathrm{FM}}$, is dependent on the applied field strength as shown in the inset to Fig. 8(a). As the applied field increases, the skyrmion diameter decreases, which results in a decreased $A_{S-}^{\mathrm{FM}}$ up to $100 \mathrm{kA} / \mathrm{m}$. However, at the same time the smaller skyrmions are more susceptible to thermally activated collapse, and thus further increase in the applied magnetic field requires increasingly larger $A_{S-}^{\mathrm{FM}}$ to maintain the same mean skyrmion creation rate.

\section{CONCLUSIONS}

Here we have studied the skyrmion creation process due to ultrafast laser pulses in thin antiferromagnetic and ferromagnetic thin films with strong interfacial DM exchange interaction. Using linearly polarized laser pulses which heat the materials above the phase transition temperature, we have shown the creation of skyrmions is probabilistic, following a Poisson counting distribution with mean rate dependent on the area heated above the phase transition temperature. This is required in order to reach a quenched magnetization state where nucleation of reversed domains can occur, which further coalesce and give rise to topological objects as the temperature decreases below the phase transition temperature. In both cases a large population of topological objects is created, including not only skyrmions, but also skyrmioniums, and more generally skyrmion bags, and domain wall skyrmions. While no domain wall skyrmions were found to survive beyond $1 \mathrm{~ns}$ after the initial laser pulses, owing to a rapid thermally activated decay, they were shown to be particularly important in controlling interactions between skyrmions, through pair annihilations between domain wall skyrmions and skyrmions with opposite topological charge. Moreover, even when a train of circularly polarized pulses is used, the probability of creating a domain wall skyrmion with a skyrmion pair cannot be disentangled from the probability of creating a single skyrmion, which prevents deterministic creation of a single skyrmion.

\section{APPENDIX A: TWO-SUBLATTICE STOCHASTIC LANDAU-LIFSHITZ-BLOCH MODEL}

We use a two-sublattice stochastic Landau-LifshitzBloch (sLLB) equation, based on the LLB equation from Refs. [71,72], extending the one-lattice LLB model [73-76], applicable for antiferromagnetic, ferrimagnetic, as well as binary ferromagnetic alloys. Similar models were also used in previous works [77-79]. We include both homogeneous and nonhomogeneous [80] interlattice exchange contributions, and recast the model in terms of accessible micromagnetic parameters, above and below the phase transition temperature. The explicit two-sublattice sLLB equation is given in Eq. (A1) 
in terms of the macroscopic magnetization, where we denote the 2 sublattices as $i=A, B$.

$$
\frac{\partial \mathbf{M}_{i}}{\partial t}=-\tilde{\gamma}_{i} \mathbf{M}_{i} \times \mathbf{H}_{\mathrm{eff}, i}-\tilde{\gamma}_{i} \frac{\tilde{\alpha}_{\perp, i}}{M_{i}} \mathbf{M}_{i} \times\left(\mathbf{M}_{i} \times\left(\mathbf{H}_{\mathrm{eff}, i}+\mathbf{H}_{t h, i}\right)\right)+\gamma_{i} \frac{\tilde{\alpha}_{\|, i}}{M_{i}}\left(\mathbf{M}_{i} . \mathbf{H}_{\|, i}\right) \mathbf{M}_{i}+\eta_{t h, i} \quad(i=A, B) .
$$

The reduced gyromagnetic ratio is given by $\tilde{\gamma}_{i}=\gamma_{i} /\left(1+\tilde{\alpha}_{\perp, i}^{2}\right)$, and the reduced transverse and longitudinal damping parameters by $\tilde{\alpha}_{\perp(\|), i}=\alpha_{\perp(\|), i} / m_{i}$, where $m_{i}(T)=M_{i}(T) / M_{S, i}^{0}$, with $M_{S, i}^{0}$ denoting the zero-temperature saturation magnetization, and $M_{i} \equiv\left|\mathbf{M}_{i}\right|$. The damping parameters are continuous at $T_{N}$-the phase transition temperature--and given by

$$
\begin{aligned}
& \alpha_{\perp, i}=\alpha_{i}\left(1-\frac{T}{3\left(\tau_{i}+\tau_{i j} m_{e, j} / m_{e, i}\right) \tilde{T}_{N}}\right), \quad T<T_{N} \\
& \alpha_{\|, i}=\alpha_{i}\left(\frac{2 T}{3\left(\tau_{i}+\tau_{i j} m_{e, j} / m_{e, i}\right) \tilde{T}_{N}}\right), \quad T<T_{N} \\
& \alpha_{\perp, i}=\alpha_{\|, i}=\frac{2 T}{3 T_{N}}, \quad T \geqslant T_{N} .
\end{aligned}
$$

We denote $\tilde{T}_{N}$ the renormalized transition temperature, given by

$$
\tilde{T}_{N}=\frac{2 T_{N}}{\tau_{A}+\tau_{B}+\sqrt{\left(\tau_{A}-\tau_{B}\right)^{2}+4 \tau_{A B} \tau_{B A}}} .
$$

The micromagnetic parameters $\tau_{i}$ and $\tau_{i j} \in[0,1]$, are coupling parameters between exchange constants and the phase transition temperature, such that $\tau_{A}+\tau_{B}=1$ and $|J|=3 \tau k_{B} T_{N}$. Here $J$ is the exchange constant for intralattice $(i=A, B)$ and interlattice $(i, j=A, B, i \neq j)$ coupling, respectively. For a simple antiferromagnet we have $\tau_{A}=\tau_{B}=\tau_{A B}=\tau_{B A}=0.5$. The normalized equilibrium magnetization functions $m_{e, i}$ are obtained from the Curie-Weiss law as

$$
m_{e, i}=B\left[\left(m_{e, i} \tau_{i}+m_{e, j} \tau_{i j}\right) 3 \tilde{T}_{N} / T+\mu_{i} \mu_{0} H_{\mathrm{ext}} / k_{B} T\right],
$$

where $B(x)=\operatorname{coth}(x)-1 / x$, and $\mu_{i}$ is the atomic magnetic moment. The magnetization length is not constant, and can differ from the equilibrium magnetization length, giving rise to a longitudinal relaxation field which includes both intralattice and interlattice contributions:

$$
\begin{aligned}
\mathbf{H}_{\|, i} & =\left\{\frac{1}{2 \mu_{0} \tilde{\chi}_{\|, i}}\left(1-\frac{m_{i}^{2}}{m_{e, i}^{2}}\right)+\frac{3 \tau_{i j} k_{B} T_{N}}{2 \mu_{0} \mu_{i}}\left[\frac{\tilde{\chi}_{\|, j}}{\tilde{\chi}_{\|, i}}\left(1-\frac{m_{i}^{2}}{m_{e, i}^{2}}\right)-\frac{m_{e, j}}{m_{e, i}}\left(\hat{\mathbf{m}}_{i} \cdot \hat{\mathbf{m}}_{j}\right)\left(1-\frac{m_{j}^{2}}{m_{e, j}^{2}}\right)\right]\right\} \mathbf{m}_{i}, \quad T<T_{N} \\
\mathbf{H}_{\|, i} & =-\left\{\frac{1}{\mu_{0} \tilde{\chi}_{\|, i}}+\frac{3 \tau_{i j} k_{B} T_{N}}{\mu_{0} \mu_{i}}\left[\frac{\tilde{\chi}_{\|, j}}{\tilde{\chi}_{\|, i}}-\frac{m_{e, j}}{m_{e, i}}\left(\hat{\mathbf{m}}_{i} \cdot \hat{\mathbf{m}}_{j}\right)\right]\right\} \mathbf{m}_{i}, \quad T>T_{N} .
\end{aligned}
$$

Here $\hat{\mathbf{m}}_{i}=\mathbf{m}_{i} / m_{i}$, and the relative longitudinal susceptibility is $\tilde{\chi}_{\|, i}=\chi_{\|, i} / \mu_{0} M_{S, i}^{0}$, where

$$
k_{B} T \tilde{\chi}_{\|, i}=\frac{\mu_{i} B_{i}^{\prime}\left(1-3 \tau_{j} \tilde{T}_{N} B_{j}^{\prime} / T\right)+\mu_{j} 3 \tau_{i j} \tilde{T}_{N} B_{i}^{\prime} B_{j}^{\prime} / T}{\left(1-3 \tau_{i} \tilde{T}_{N} B_{i}^{\prime} / T\right)\left(1-3 \tau_{j} \tilde{T}_{N} B_{j}^{\prime} / T\right)-\tau_{i j} \tau_{j i} B_{i}^{\prime} B_{j}^{\prime}\left(3 \tilde{T}_{N} / T\right)^{2}},
$$

and $B_{i}^{\prime} \equiv B_{m_{e, i}}^{\prime}\left[\left(m_{e, i} \tau_{i}+m_{e, j} \tau_{i j}\right) 3 \tilde{T}_{N} / T\right]$

The effective field in Eq. (A1) is a sum of all the interaction fields, and given by $\mathbf{H}_{\mathrm{eff}, i}=\mathbf{H}_{\mathrm{ext}, i}+\mathbf{H}_{\mathrm{demag}, i}+\mathbf{H}_{\mathrm{ani}, i}+\mathbf{H}_{\mathrm{ex}, i}$. In particular $\mathbf{H}_{\text {demag, } i}$ is the demagnetizing field calculated for the net magnetization $\left(\mathbf{M}_{A}+\mathbf{M}_{B}\right) / 2$, and applied equally to both sublattices. The uniaxial anisotropy field is given by

$$
\mathbf{H}_{\mathrm{ani}, i}=\frac{2 K_{1, i}}{\mu_{0} M_{e, i}^{2}}\left(\mathbf{M}_{i} \cdot \mathbf{e}_{A}\right) \mathbf{e}_{A} .
$$

Here $\mathbf{e}_{A}$ is the symmetry axis, $M_{e, i}=m_{e, i} M_{S, i}^{0}$, and $K_{1, i}$ follows the temperature dependence $K_{1, i}=K_{1, i}^{0} m_{e, i}^{3}$. The exchange field includes both the isotropic direct exchange term, as well as the interfacial DMI exchange term. The direct exchange term includes the usual intralattice contribution, as well as homogeneous and nonhomogeneous interlattice contributions, and is given by

$$
\mathbf{H}_{\mathrm{ex}, i}=\frac{2 A_{i}}{\mu_{0} M_{e, i}^{2}} \nabla^{2} \mathbf{M}_{i}+\frac{4 A_{h, i}}{\mu_{0} M_{e, i} M_{e, j}} \mathbf{M}_{j}+\frac{A_{n h, i}}{\mu_{0} M_{e, i} M_{e, j}} \nabla^{2} \mathbf{M}_{j}
$$

The intralattice exchange stiffness $A_{i}$ has the temperature dependence $A_{i}=A_{i}^{0} m_{e, i}^{2}$, while the interlattice exchange stiffnesses have the temperature dependences $A_{h(n h), i}=A_{h(n h), i}^{0} m_{e, i} m_{e, j}$. The interfacial DMI exchange field is given by, applicable for systems with $C_{n v}$ symmetry,

$$
\mathbf{H}_{i \mathrm{DMI}, i}=-\frac{2 D_{i}}{\mu_{0} M_{e, i}^{2}}\left(\frac{\partial M_{z}}{\partial x}, \frac{\partial M_{z}}{\partial y},-\frac{\partial M_{x}}{\partial x}-\frac{\partial M_{y}}{\partial y}\right),
$$

with the DMI exchange parameter having the temperature dependence $D_{i}=D_{i}^{0} m_{e, i}^{2}$. 
Finally, the terms $\mathbf{H}_{t h, i}$ and $\boldsymbol{\eta}_{t h, i}$ are stochastic quantities with zero spatial, vector components, and interlattice correlations, and whose components follow Gaussian distributions with zero mean and standard deviations given, respectively, by

$$
\begin{aligned}
H_{t h, i}^{\text {std. }} & =\frac{1}{\alpha_{\perp, i}} \sqrt{\frac{2 k_{B} T\left(\alpha_{\perp, i}-\alpha_{\|, i}\right)}{\gamma_{i} \mu_{0} M_{S, i}^{0} V \Delta t}} \\
\eta_{t h, i}^{\text {std. }} & =\sqrt{\frac{2 k_{B} T \alpha_{\|, i} \gamma_{i} M_{S, i}^{0}}{\mu_{0} V \Delta t}} .
\end{aligned}
$$

Here $V$ is the stochastic computational cell-size volume, and $\Delta t$ is the integration time step. Similar to the approach in Ref. [76], it can be shown the magnetization length distribution follows a Boltzmann probability distribution. For the twosublattice case, in general this distribution is a function of the magnetization of both sublattices, $m_{A}$ and $m_{B}$, and is shown below for the isotropic case:

$$
P_{i}\left(m_{A}, m_{B}\right) \propto m_{i}^{2} \exp \left\{-\frac{M_{S}^{0} V}{4 \mu_{i} m_{e, i} k_{B} T}\left[\frac{\left(m_{i}^{2}-m_{e, i}^{2}\right)^{2}}{m_{e, i}} \frac{\left(\mu_{i}+3 \tau_{i j} k_{B} T_{N} \tilde{\chi}_{\|, j}\right)}{2 \tilde{\chi}_{\|, i}}+\frac{\left(m_{j}^{2}-m_{e, j}^{2}\right)}{m_{e, j}} 3 \tau_{i j} k_{B} T_{N} m_{i}^{2}\right]\right\} .
$$

Verification of Eq. (A11) is given in Ref. [54].

The temperature is solved using a two-temperature model, where the electron and lattice temperature are coupled using rate equations as shown in Eq. (A12). The magnetization is coupled to the electron bath via the Landau-Lifshitz damping, thus in the LLB equation we have $T=T_{e}$.

$$
\begin{aligned}
C_{e} \rho \frac{\partial T_{e}(\mathbf{r}, t)}{\partial t} & =\nabla \cdot K \nabla T_{e}-G_{e}\left(T_{e}-T_{l}\right)+S \\
C_{l} \rho \frac{\partial T_{l}}{\partial t} & =G_{e}\left(T_{e}-T_{l}\right) .
\end{aligned}
$$

In Eq. (A12) $C_{e}$ and $C_{l}$ are, respectively, the electron and lattice specific heat capacities, $\rho$ is the mass density, $K$ is the thermal conductivity, and $G_{e}$ is the electron-lattice coupling constant, typically on the order $10^{18} \mathrm{~W} / \mathrm{m}^{3} \mathrm{~K}$.

\section{APPENDIX B: SIMULATION PARAMETERS}

Simulations were performed using GPU-accelerated computations, using the finite-difference formulation of the twosublattice sLLB model coupled to the two-temperature model. The code used for this work is open source and available at [81,82]. The cell size was set to $1 \times 1 \times 2 \mathrm{~nm}^{3}$ and thin films were simulated by employing periodic boundary conditions for the demagnetizing field and differential operators. The magnetization dynamics were solved using the Heun method with a time step of 0.5 to $1 \mathrm{fs}$ during the ultrafast demagnetization stage and 1 to $5 \mathrm{fs}$ during the longer magnetization recovery stage. For the AFM thin film, material parameters were used as: $\alpha=0.1, M_{S}^{0}=400 \mathrm{kA} / \mathrm{m}, A=5 \mathrm{pJ} / \mathrm{m}, K_{1}=100 \mathrm{~kJ} / \mathrm{m}^{3}$ with easy axis perpendicular to the film, $A_{h} / a^{3}=-10 \mathrm{MJ} / \mathrm{m}^{3}$ with $a$ the lattice constant, $A_{n h}=-10 \mathrm{pJ} / \mathrm{m}, D=1 \mathrm{~mJ} / \mathrm{m}^{2}$, and $T_{N}=500 \mathrm{~K}$. For the FM thin film we used: $\alpha=0.1, M_{S}^{0}=600 \mathrm{kA} / \mathrm{m}, A=10 \mathrm{pJ} / \mathrm{m}, K_{1}=380 \mathrm{~kJ} / \mathrm{m}^{3}$ with easy axis perpendicular to the film, $D=-1.5 \mathrm{~mJ} / \mathrm{m}^{2}$, and $T_{C}=500 \mathrm{~K}$. For the two-temperature model we used $C_{e}=40 \mathrm{~J} / \mathrm{kgK}, C_{l}=130 \mathrm{~J} / \mathrm{kgK}, K=147 \mathrm{~W} / \mathrm{mK}$, $\rho=22650 \mathrm{~kg} / \mathrm{m}^{3}$.

[1] I. Dzyaloshinsky, J. Phys. Chem. Solids 4, 241 (1958).

[2] T. Moriya, Phys. Rev. 120, 91 (1960).

[3] A. M. Kosevich, B. A. Ivanov, and A. S. Kovalev, Phys. Rep. 194, 117 (1990).

[4] A. N. Bogdanov and D. Yablonskii, Zh. Eksp. Teor. Fiz. 95, 178 (1989) [Sov. Phys. JETP 68, 101 (1989)].

[5] S. Mühlbauer, B. Binz, F. Jonietz, C. Pfleiderer, A. Rosch, A. Neubauer, R. Georgii, and P. Böni, Science 323, 915 (2009).

[6] X. Z. Yu, Y. Onose, N. Kanazawa, J. H. Park, J. H. Han, Y. Matsui, N. Nagaosa, and Y. Tokura, Nature (London) 465, 901 (2010).

[7] C. Moreau-Luchaire, C. Moutafis, N. Reyren, J. Sampaio, C. A. F. Vaz, N. Van Horne, K. Bouzehouane, K. Garcia,
C. Deranlot, P. Warnicke et al., Nat. Nanotechnol. 11, 444 (2016).

[8] O. Boulle, J. Vogel, H. Yang, S. Pizzini, D. de Souza Chaves, A. Locatelli, T. Onur Menteş, A. Sala, L. D. Buda-Prejbeanu, O. Klein et al., Nat. Nanotechnol. 11, 449 (2016).

[9] N. Nagaosa and Y. Tokura, Nat. Nanotechnol. 8, 899 (2013).

[10] S. Woo, K. Litzius, B. Krüger, M.-Y. Im, L. Caretta, K. Richter, M. Mann, A. Krone, R. M. Reeve, M. Weigand et al., Nat. Mater. 15, 501 (2016).

[11] W. Legrand, D. Maccariello, N. Reyren, K. Garcia, C. Moutafis, C. Moreau-Luchaire, S. Collin, K. Bouzehouane, V. Cros, and A. Fert, Nano Lett. 17, 2703 (2017). 
[12] W. Jiang, X. Zhang, G. Yu, W. Zhang, X. Wang, M. B. Jungfleisch, J. E. Pearson, X. Cheng, O. Heinonen, K. L. Wang et al., Nat. Phys. 13, 162 (2017).

[13] J. Sampaio, V. Cros, S. Rohart, A. Thiaville, and A. Fert, Nat. Nanotechnol. 8, 839 (2013).

[14] S. Lepadatu, Sci. Rep. 9, 9592 (2019).

[15] J. Barker and O. A. Tretiakov, Phys. Rev. Lett. 116, 147203 (2016).

[16] E. G. Galkina and B. A. Ivanov, Low Temp. Phys. 44, 618 (2018).

[17] T. Jungwirth, X. Marti, P. Wadley, and J. Wunderlich, Nat. Nanotechnol. 11, 231 (2016).

[18] A. K. Nayak, V. Kumar, T. Ma, P. Werner, E. Pippel, R. Sahoo, F. Damay, U. K. Rößler, C. Felser, and S. S. P. Parkin, Nature (London) 548, 561 (2017).

[19] J. Jena, R. Stinshoff, R. Saha, A. K. Srivastava, T. Ma, H. Deniz, P. Werner, C. Felser, and S. S. P. Parkin, Nano Lett. 20, 59 (2020).

[20] A. Bogdanov and A. Hubert, J. Magn. Magn. Mater. 195, 182 (1999).

[21] D. Foster, C. Kind, P. J. Ackerman, J.-S. B. Tai, M. R. Dennis, and I. I. Smalyukh, Nat. Phys. 15, 655 (2019).

[22] V. I. Fal'ko and S. V. Iordanskii, Phys. Rev. Lett. 82, 402 (1999).

[23] P. Jennings and P. Sutcliffe, J. Phys. A: Math. Theor. 46, 465401 (2013).

[24] S. B. Gudnason and M. Nitta, Phys. Rev. D 89, 085022 (2014).

[25] R. Cheng, M. Li, A. Sapkota, A. Rai, A. Pokhrel, T. Mewes, C. Mewes, D. Xiao, M. De Graef, and V. Sokalski, Phys. Rev. B 99, 184412 (2019).

[26] M. Li, A. Sapkota, A. Rai, A. Pokhrel, T. Mewes, C. Mewes, D. Xiao, M. De Graef, and V. Sokalski, arXiv:2004.07888.

[27] E. Beaurepaire, J.-C. Merle, A. Daunois, and J.-Y. Bigot, Phys. Rev. Lett. 76, 4250 (1996).

[28] B. Koopmans, G. Malinowski, F. D. Longa, D. Steiauf, M. Fähnle, T. Roth, M. Cinchetti, and M. Aeschlimann, Nat. Mater. 9, 259 (2010).

[29] W. H. Zurek, Phys. Rep. 276, 177 (1996).

[30] P. C. Hendry, N. S. Lawson, R. A. M. Lee, P. V. E. McClintock, and C. D. H. Williams, Nature (London) 368, 315 (1994).

[31] I. Chuang, R. Durrer, N. Turok, and B. Yurke, Science 251, 1336 (1991).

[32] C. D. Stanciu, F. Hansteen, A. V. Kimel, A. Kirilyuk, A. Tsukamoto, A. Itoh, and Th. Rasing, Phys. Rev. Lett. 99, 047601 (2007).

[33] A. Kirilyuk, A. V. Kimel, and T. Rasing, Rev. Mod. Phys. 82, 2731 (2010).

[34] A. R. Khorsand, M. Savoini, A. Kirilyuk, A. V. Kimel, A. Tsukamoto, A. Itoh, and Th. Rasing, Phys. Rev. Lett. 108, 127205 (2012).

[35] T. A. Ostler, J. Barker, R. F. L. Evans, R. W. Chantrell, U. Atxitia, O. Chubykalo-Fesenko, S. El Moussaoui, L. Le Guyader, E. Mengotti, L. J. Heyderman et al., Nat. Commun. 3, 666 (2012).

[36] I. Radu, K. Vahaplar, C. Stamm, T. Kachel, N. Pontius, H. A. Dürr, T. A. Ostler, J. Barker, R. F. L. Evans, R. W. Chantrell et al., Nature (London) 472, 205 (2011).

[37] C.-H. Lambert, S. Mangin, B. S. D. Ch. S. Varaprasad, Y. K. Takahashi, M. Hehn, M. Cinchetti, G. Malinowski, K. Hono, Y. Fainman, M. Aeschlimann, and E. E. Fullerton, Science 345, 1337 (2014).
[38] S. Mangin, M. Gottwald, C.-H. Lambert, D. Steil, V. Uhlî́, L. Pang, M. Hehn, S. Alebrand, M. Cinchetti, G. Malinowski et al., Nat. Mater. 13, 286 (2014).

[39] R. B. Wilson, J. Gorchon, Y. Yang, C.-H. Lambert, S. Salahuddin, and J. Bokor, Phys. Rev. B 95, 180409(R) (2017).

[40] N. Bergeard, M. Hehn, S. Mangin, G. Lengaigne, F. Montaigne, M. L. M. Lalieu, B. Koopmans, and G. Malinowski, Phys. Rev. Lett. 117, 147203 (2016).

[41] M. L. M. Lalieu, R. Lavrijsen, and B. Koopmans, Nat. Commun. 10, 110 (2019).

[42] M. Finazzi, M. Savoini, A. R. Khorsand, A. Tsukamoto, A. Itoh, L. Duò, A. Kirilyuk, Th. Rasing, and M. Ezawa, Phys. Rev. Lett. 110, 177205 (2013).

[43] T. Ogasawara, N. Iwata, Y. Murakami, H. Okamoto, and Y. Tokura, Appl. Phys. Lett. 94, 162507 (2009).

[44] G. Berruto, I. Madan, Y. Murooka, G. M. Vanacore, E. Pomarico, J. Rajeswari, R. Lamb, P. Huang, A. J. Kruchkov, Y. Togawa et al., Phys. Rev. Lett. 120, 117201 (2018).

[45] S.-G. Je, P. Vallobra, T. Srivastava, J.-C. Rojas-Sánchez, T. H. Pham, M. Hehn, G. Malinowski, C. Baraduc, S. Auffret, G. Gaudin et al., Nano Lett. 18, 7362 (2018).

[46] O. P. Polyakov, I. A. Gonoskov, V. S. Stepanyuk, and E. K. U. Gross, J. Appl. Phys. 127, 073904 (2020).

[47] H. Fujita and M. Sato, Phys. Rev. B 95, 054421 (2017).

[48] R. Khoshlahni, A. Qaiumzadeh, A. Bergman, and A. Brataas, Phys. Rev. B 99, 054423 (2019).

[49] W. Koshibae and N. Nagaosa, Nat. Commun. 5, 5148 (2014).

[50] U. Atxitia and O. Chubykalo-Fesenko, Appl. Phys. Lett. 91, 232507 (2007).

[51] U. Atxitia and O. Chubykalo-Fesenko, Phys. Rev. B 84, 144414 (2011).

[52] J. Mendil, P. Nieves, O. Chubykalo-Fesenko, J. Walowski, T. Santos, S. Pisana, and M. Münzenberg, Sci. Rep. 4, 3980 (2014).

[53] S. Lepadatu, J. Appl. Phys. 120, 163908 (2016).

[54] See Supplemental Material at http://link.aps.org/supplemental/ 10.1103/PhysRevB.102.094402 for further details on LandauLifshitz-Bloch model.

[55] M. Labrune, S. Andrieu, F. Rio, and P. Bernstein, J. Magn. Magn. Mater. 80, 211 (1989).

[56] J. Pommier, P. Meyer, G. Pénissard, J. Ferré, P. Bruno, and D. Renard, Phys. Rev. Lett. 65, 2054 (1990).

[57] V. G. Bar'yakhtar, Zh. Eksp. Teor. Fiz. 87, 1501 (1984) [Sov. Phys. JETP 60, 863 (1984)].

[58] K. Vahaplar, A. M. Kalashnikova, A. V. Kimel, S. Gerlach, D. Hinzke, U. Nowak, R. Chantrell, A. Tsukamoto, A. Itoh, A. Kirilyuk, and Th. Rasing, Phys. Rev. B 85, 104402 (2012).

[59] E. Iacocca, T.-M. Liu, A. H. Reid, Z. Fu, S. Ruta, P. W. Granitzka, E. Jal, S. Bonetti, A. X. Gray, C. E. Graves et al., Nat. Commun. 10, 1756 (2019).

[60] S. Z. Lin, C. Reichhardt, C. D. Batista, and A. Saxena, Phys. Rev. B 87, 214419 (2013).

[61] S. Rohart, J. Miltat, and A. Thiaville, Phys. Rev. B 93, 214412 (2016).

[62] D. Cortés-Ortuño, W. Wang, M. Beg, R. A. Pepper, M.-A. Bisotti, R. Carey, M. Vousden, T. Kluyver, O. Hovorka, and H. Fangohr, Sci. Rep. 7, 4060 (2017).

[63] A. Derras-Chouk, E. M. Chudnovsky, and D. A. Garanin, J. Appl. Phys. 126, 083901 (2019). 
[64] P. F. Bessarab, D. Yudin, D. R. Gulevich, P. Wadley, M. Titov, and O. A. Tretiakov, Phys. Rev. B 99, 140411(R) (2019).

[65] J. Wild, Sci. Adv. 3, e1701704 (2017).

[66] R. Medapalli, D. Afanasiev, D. K. Kim, Y. Quessab, S. Manna, S. A. Montoya, A. Kirilyuk, Th. Rasing, A. V. Kimel, and E. E. Fullerton, Phys. Rev. B 96, 224421 (2017).

[67] M. S. El Hadri, P. Pirro, C.-H. Lambert, S. Petit-Watelot, Y. Quessab, M. Hehn, F. Montaigne, G. Malinowski, and S. Mangin, Phys. Rev. B 94, 064412 (2016).

[68] V. Raposo, R. Guedas, F. García-Sánchez, M. A. Hernández, M. Zazo, and E. Martínez, Appl. Sci. 10, 1307 (2020).

[69] M. S. El Hadri, M. Hehn, P. Pirro, C.-H. Lambert, G. Malinowski, E. E. Fullerton, and S. Mangin, Phys. Rev. B 94, 064419 (2016).

[70] See Supplemental Material at http://link.aps.org/supplemental/ 10.1103/PhysRevB.102.094402 for skyrmion creation statistics as a function of DMI strength.

[71] U. Atxitia, P. Nieves, and O. Chubykalo-Fesenko, Phys. Rev. B 86, 104414 (2012).
[72] P. Nieves, U. Atxitia, R. W. Chantrell, and O. ChubykaloFesenko, Low Temp. Phys. 41, 739 (2015).

[73] D. A. Garanin, Phys. Rev. B 55, 3050 (1997).

[74] O. Chubykalo-Fesenko, U. Nowak, R. W. Chantrell, and D. A. Garanin, Phys. Rev. B 74, 094436 (2006).

[75] D. A. Garanin and O. Chubykalo-Fesenko, Phys. Rev. B 70, 212409 (2004).

[76] R. F. L. Evans, D. Hinzke, U. Atxitia, U. Nowak, R. W. Chantrell, and O. Chubykalo-Fesenko, Phys. Rev. B 85, 014433 (2012).

[77] M. Menarini, R. Medapalli, E. E. Fullerton, and V. Lomakin, AIP Adv. 9, 035040 (2019).

[78] V. Puliafito, R. Khymyn, M. Carpentieri, B. Azzerboni, V. Tiberkevich, A. Slavin, and G. Finocchio, Phys. Rev. B 99, 024405 (2019).

[79] C. Vogler, C. Abert, F. Bruckner, and D. Suess, Phys. Rev. B 100, 054401 (2019).

[80] L. Sánchez-Tejerina, V. Puliafito, P. Khalili Amiri, M. Carpentieri, and G. Finocchio, Phys. Rev. B 101, 014433 (2020).

[81] https://github.com/SerbanL/Boris2.

[82] S. Lepadatu, arXiv:2008.02478. 